\title{
A passion for development
}

\author{
The Coiled Spring: How Life Begins \\ by Ethan Bier \\ Cold Spring Harbour Laboratory Press · August 2000 \\ Paperback $\mathfrak{E 3 1 / \$ 3 9}$
}

Maria Leptin and Siegfried Roth

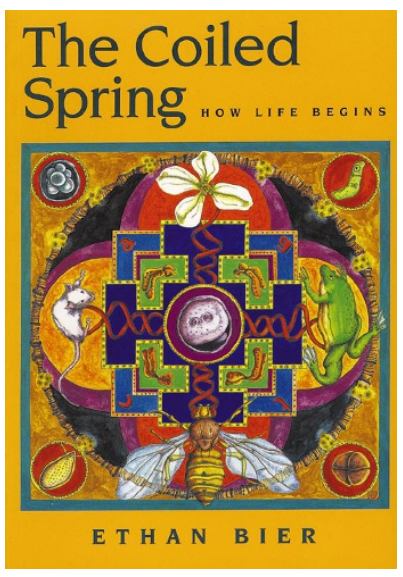

$\int$ riven by genetics and the application of molecular biology, the understanding of the development of organisms has made dramatic progress over the past two decades. The principles of patterning the early embryo are now understood for Drosophila and are emerging for other species. The growth and patterning of appendages have been solved, and processes such as differentiation or cell death are now understood in impressive detail. Many developmental biologists must have contemplated conveying their excitement over the revolutionary discoveries and the continuous stream of eye-opening results to a wider audience. Indeed, in addition to classic developmental biology textbooks being re-edited and new ones being written, several more idiosyncratic, often even personal, accounts of this field of biology have been published.

The framework for Ethan Bier's view of development as presented in this book is provided by the generalizations that have emerged in the recent past. He does not try to cover all of development, nor does he concentrate on one organism. Instead, he chooses two processes whose basic principles are well understood-the establishment of the primary axes in the embryo, and the patterning and growth of appendagesand describes in six chapters how they work in fruitflies, in vertebrates and in plants. This choice, which excludes large areas of developmental biology, is based on one of the most amazing discoveries of the past decade; that of the extensive parallels between developmental mechanisms and their molecular bases in distant phyla. Arms in vertebrates and wings of flies, or eyes in all species, are after all built according to the same principles from the same moleculesa big surprise and a challenge to the textbook dogma that there is no homology between insect and vertebrate appendages.

These six chapters are the core of the book. For the non-biologist, two introductory chapters explain in simple language and clear logic the principles of genetics and molecular biology needed to under-

stand all details of the six core chapters. Interspersed within the main text, Bier includes 17 'bioboxes'. These summaries of the lives of scientists who have made important contributions to developmental biology leave one with the conviction that science is not a dry, objective matter but is as full of life and excitement as the people who do it. The discussion of what, in their own views, made these scientists what they are, and what it takes to do good science, should be read by every graduate student (and then re-read as a postdoctoral worker, and then as a professor).

Bier has singled out pattern formation as the topic for his book for good reasons. It would nevertheless have been desirable to see some reference to other areas of development not touched upon at all in this book, and especially to the remaining unsolved or partly solved questions such as the problem of patterning within a single cell, or that of the determination of the size and shape of an organism. A discussion of these might have tempered his optimism in predicting "In any case it should be possible from first principles to design an animal that at least looks like a T-Rex". That may be true in the long run, but it would have been interesting to indicate the types of intellectual challenges that we face before we can accomplish this.

Bier clearly loves explaining science and cares that everyone should be able to understand his story. He uses several tricks to help both the non-specialist and the biologically trained reader along. Above all, he enjoys finding striking metaphors to describe complex phenomena (for example, he compares genes in cells to electric appliances in a kitchen, which are on and off in different combinations depending on the task being done in the kitchen). The text is peppered with such metaphors, which makes for entertaining reading and does help to get the important points across.

Bier has aimed high in trying to address the general science reader. For the non-biologist some parts are too detailed, which cannot be disguised by amusing analogies and metaphors. In contrast, one's enthusiasm for recommending the book to readers to whom not just the story but the actual facts are important is marred by a deluge of inaccuracies and mistakes. Some arise from oversimplifications ('skin' may be simpler than 'epidermis', but that is not what insect embryos have), others from relying on hearsay rather than careful research. For example, the name for the gene decapentaplegic, one central to Bier's own work, alludes to the loss of all 15 (dekapenta in Greek) imaginal disks, not to some 50 things in general going wrong. These are not major problems; others are more painful, especially where they concern the historical record on subjects not usually found in textbooks, so that readers will not necessarily see the account found here put right elsewhere. Although it has become fashionable to deny Spemann's role in the discovery of the organizer and to give all credit to Mangold - and it is therefore not surprising to see this version of history reiterated here (Mangold gets a biobox, but Spemann doesn't-good accounts that attest to the contrary do exist. And although many theoreticians in the 1980 s subscribed to the view that segments were created by chemical waves, it is unfair to quote Hans Meinhard for this, because he was probably the first to realize that such models cannot account for the phenotypes of the newly discovered segmentation genes and instead proposed a model that emphasized the hierarchical and combinatorial control of individual segments.

However, nit-picking misses the point of the bookWe should simply hope for so much popularity for the book that Bier will have to write a revised second edition, which should add a little more scientific precision while retaining both the simplicity and the enthusiasm.

Maria Leptin and Siegfried Roth are at the Institute of Genetics and Institute of Developmental Biology, University of Cologne, Germany

email:mleptin@uni-koeln.de 